\title{
Pseudo endocrine patient
}

\section{Editorial}

On the AACE Conference may 2017 in Austin Texas, Dr. Michael T McDermott MD Professor of Medicine, University of Colorado Denver School of Medicine had presented a daily problem in our Adult Endocrinology practice, it is a false impression by some group of patients as they have hormonal disorders especially related in majority to the thyroid, adrenal and occasionally to the pituitary, this group has been given the title of (Pseudo Endocrine Patient), on his talk Dr. McDermott had delivered a very sound perspective about understanding the spectrum of signs and symptoms that the patient presented and the correlation with hormonal assay and put very practical approach to Pseudo Endocrine Patient, and to consider this particular patient intentions and interest in following social media and huge variety of for medical, pharmaceutical and commercial websites at the internet.

He had delivered his interesting lecture supported by cases from representing patient from this category of presumably thyroidal illness, (please open the first link down) where you can go through those cases and comprehensive and interactive discussion and the sound deduction taken for management.

I have received many cases referred by practitioners, surgeons, gynecologists and internists of hypercortisolemia with high or normal ACTH with presumptive diagnosis of Cushing's syndrome, after my through clinical, physical examinations and biochemical work-up I have found most those cases are (Pseudo-Cushing Syndrome), the question that might be asked why such good number of referred cases while Cushing's syndrome is a rare disorder and what are the external physical features that made the diagnosis of Cushing by the referring doctors.

The following non adrenal illnesses by order of frequency:

a. Obesity

b. Depressive illness

c. PCOS

d. Chronic alcoholism

e. Obstructive Sleep Apnea

f. High Total Cortisol, conditions of high SHBP, birth pills...

g. Other conditions like eating disorders, malnutrition had been mentioned in the literature but not in our cases so far!

Those patients have shared some of the physical features of genuine Cushing, but interestingly I have found patient determination of been ill with adrenal problem, and they confidently support their arguments by abnormal Biochemical results! Therefore we can consider them as (pseudo endocrine patient) and precisely pseudo Cushing's syndrome

Cushing's syndrome is a rare disorder which can severely affect the patient. Symptoms of patients with Cushing's syndrome include weight gain, easy skin and subcutaneous bruising, typical purplish wide skin striae menstrual irregularities, increased appetite, insomnia, depression or mood fluctuations, anxiety, fatigue, agitation and altered mentation and in extreme case frank psychosis, bone pains
Volume 6 Issue 3 - 2018

\author{
Saadi JS AlJadir \\ Ministry of Higher Education \& Scientific Research, Iraq
}

Correspondence: Saadi JS AlJadir, Ministry of Higher Education \& Scientific Research, PO Box 498, Nassiryia, ThiQar, Iraq, Email saljadir5I@gmail.com

Received: February 26, 2018 | Published: June 14, 2018

and occasionally fragility fractures Physical abnormalities include new onset obesity (primarily in the abdominal and buttock regions), buffalo hump, filling in of the regions above the collarbone, thinning of the extremities, the face is rounded and plethoric, acne, thin skin, decreased muscle strength and proximal myopathy, high blood pressure, stretch marks and hirsutism in females. Cushing's syndrome remains one of the most challenging endocrine pathologies.

Most clinical features overlap with those of common diseases found in the general population (Pseudo Cushing), and some patients have an atypical clinical presentation with only isolated symptoms.

\section{Pseudo cushing's}

A pseudo-Cushing's state may be defined as some or all of the clinical features that resemble true Cushing's syndrome together with biochemical evidence of hypercortisolism. It is a particular type of hypercortisolism presenting during the above conditions, named functional hypercortisolism, is caused by chronic activation of hypothalamic-pituitary-adrenal (HPA) axis, through hypothalamic or supra hypothalamic stimulation of the axis . It is usually mild and disappears when the underlying disorder is resolved. PseudoCushing's syndrome is not caused by a disease of the hypothalamicpituitary-adrenal axis as Cushing's is.

\section{Metabolic syndrome}

Obesity is increasing in general population all around the globe and ranking high in some developing countries, besides the enormous changes in dietary habits with remarkable increase in sugars and fat consumptions, there is scarcity in daily physical activity and lack of physical games in this region. ${ }^{1-5}$

Truncal Obesity is characteristic of metabolic syndrome that shares many physical features of genuine hypercortisolism, with almost similar fat distribution and skin striae (although not typical for expert look!), hypertension, glucose intolerance, diabetes, dyslipidemia, insulin resistance and hyperinsulinemia, although cushingoid facies are not seen this condition but can been seen in some alcoholics!

The differentiation between mild Cushing's syndrome and pseudoCushing's syndrome can be difficult and creates a considerable challenge to endocrinologist as cutoff ranges and false negative or positive results have induced huge confusion about diagnostic certainty, yet we have to utilize minimal battery of tests and avoid more invasive testing and stay vigilant toward patients' allegations, 
we shouldn't underestimate eruption of new signs during follow up.

Signs that differentiate Cushing's syndrome from pseudoCushingoid states most reliably include the presence of proximal myopathy, easy bruising, and thinness and fragility of the skin. Physical signs such as buffalo hump, obesity, and hirsutism are nonspecific and consider and can be shared by the two conditions.

\section{Hormonal \& biochemical work-up:}

1. Serum cortisol circadian rhythm: the diurnal rhythmicity of cortisol secretion is invariably retained in Patient of pseudo Cushing

2. Screening Test (Over-Night DST) The overnight test involves the oral administration of $0.5-2.0 \mathrm{mg}$ dexamethasone (most commonly $1 \mathrm{mg}$ ) at 2300 or $2400 \mathrm{~h}$, after which a plasma cortisol sample is obtained at $0800 \mathrm{~h}$ or $0900 \mathrm{~h}$ the next morning. The reported cut-off values for suppression of serum cortisol in studies utilizing modern RIAs, but with relatively small numbers of individuals with Cushing's syndrome, range from 100-200 nmol/liter (3.6-7.2 $\mu \mathrm{g} / \mathrm{dl})$, the standard dexamethasone suppression test is considered to minimize the false negative or positive

3. The Dex-CRH test is based on the fact that patients with $\mathrm{CD}$ can get pituitary-adrenal response after administration of $\mathrm{CRH}$ injection despite pre-treatment with dexamethasone. Patients with PCS, however, are relatively unresponsive to CRH stimulation when suppressed by dexamethasone.

4. We can conclude that the Dex-CRH test, and measure cortisol in saliva or serum collected at midnight have showed high diagnostic accuracy, however a CRH test after the standard 2-day low-dose dexamethasone suppression test has been reported to distinguish true from pseudo-Cushing's syndrome.

In normal subjects and in patients with endogenous depression, insulin-induced hypoglycemia results in a rise in ACTH and cortisol levels, a response that usually is not seen in patients with Cushing's syndrome. Finally, loperamide (opiate derivative, $8 \mathrm{mg}$ given and 3.5 hour cortisol collected) lowers cortisol values in patients with pseudoCushing but not in those with true Cushing's syndrome.

\section{Acknowledgments}

None.

\section{Conflict of interest}

The author declares there is conflict of interest

\section{References}

1. http://am2016.aace.com/presentations/thursday/T71\&T72/pseudo_ endocrine_disorders.pdf

2. Alwani RA, Schmit Jongbloed LW, de Jong FH, et al. Differentiating between Cushing's disease and pseudo-Cushing's syndrome: comparison of four tests. Eur J Endocrinol. 2014;170(4):477-86.

3. John NP, Peter T, Michael B, et al. The Diagnosis and Differential Diagnosis of Cushing's Syndrome and Pseudo-Cushing's States. Endocrine Reviews. 1998;19(5):647-672.

4. Williams Textbook of Endocrinology, 12th edition. Shlomo MelmedKenneth Polonsky P. Reed Larsen Henry Kronenberg.

5. Pseudo-Cushing Syndrome Updated. Catherine Anastasopoulou, Chief Editor: George T Griffing. Medscape, 2017 